\title{
METEOROLOGICAL FACTORS AND POLLEN SEASON DYNAMICS OF SELECTED HERBACEOUS PLANTS IN SZCZECIN, 2004-2008
}

\author{
Małgorzata Puc \\ Department of Botany and Nature Conservation, University of Szczecin, Poland \\ Felczaka 3c, 71-412 Szczecin, Poland \\ e-mail:mapuc@univ.szczecin.pl
}

Received: 9.08.2009

\section{Abstract}

The pollen of mugwort, plantain, sorrel, nettle and pigweed is an important airborne allergen source worldwide. The occurrence of pollen grains in the air is a seasonal phenomenon and estimation of seasonal variability in the pollen count permits evaluation of the threat posed by allergens over a given area. The aim of the study was to analyse the dynamics of Artemisia, Plantago, Rumex, Urticaceae and Chenopodiaceae pollen season in Szczecin (western Poland) in 2004-2008 and to establish a relationship between the meteorological parameters versus the pollen count of the taxa studied. Measurements were performed by the Hirst volumetric trap (model Lanzoni VPPS 2000). Consecutive phases during the pollen season were defined for each taxon $(1,5,25,50,75,95,99 \%$ of annual total) and duration of the season was determined using the $98 \%$ method. On the basis of this analysis, temporary differences in the dynamics of the seasons were most evident for Artemisia. Correlation analysis with weather parameters demonstrated that the maximum wind speed, mean and maximum air temperature, relative humidity and dew point are the main factors influencing the average daily pollen concentrations in the atmosphere.

Key words: aeroallergens, pollen season, seasonal variation, weather factors, Szczecin

\section{INTRODUCTION}

In the temperate climatic zone, the seasonal changes in pollen count in the air depend on the seasons of the year. The corresponding atmospheric pollen seasons are described by the start and end dates, duration of airborne pollen as well as the date and value of the maximum pollen count. Analysis of these data permits the determination of the dynamics of the pollen season and estimation of the rate of changes in the pollen count in the air relative to the annual total (S z c ze pan e k, 1994).

Aerobiological studies providing data on exposure of people to allergens in the air are of substantial importance in pollen allergy diagnosis (R a piejko et al. 2004). The most important agent causing pollinosis in Europe is herbaceous pollen. Approximate estimations indicate that in Europe 3-5\% of the population suffer from pollinosis from May to August, during the flowering season (D' A m a to and S piek s ma, 2004). In this time, high concentrations of pollen from these taxa occur also in Szczecin. Allergic respiratory disorders of a population in a given geographical region are mainly determined by the presence and quantity of native plants causing allergy. Exacerbation of the disease symptoms is seasonal and related to the presence of pollen grains in the air. The start and end dates of the pollen seasons as well as the intensity of pollen production in different regions in Europe can differ significantly over the years ( $\mathrm{Kadocsa}$ and J u h a s z, 2002).

In aerobiological studies, especially in the analysis of the dynamics of pollen seasons, meteorological conditions are used to explain the shifts in the start and end dates of the pollination seasons and in duration of particular phenophases. Weather parameters are known to affect the dispersion dynamics of particles of biological origin. The release and dispersion of pollen also depend on the microclimate, which explains the differences in the timing of flowering and occurrence of pollen in the air within the same species (Kasprzyk et al. 2004; Garcia-Mozo et al. 2007; S ilva Palacios et al. 2007).

The aim of our study was to analyse the dynamics of pollen seasons in selected taxa to evaluate the threat posed by the presence of pollen aeroallergens in the air of Szczecin and to establish relationships between pollen concentrations and climatic factors.

\section{MATERIALS AND METHODS}

Szczecin is situated in Northwest Poland. The climate of the region is modified by the influence of Atlantic air masses and the proximity of the Baltic Sea. 
Within the city area, there are synanthropic plants and trees introduced by man and also a primeval forest.

Analysis of pollen distribution was performed in Szczecin in the seasons of 2004-2008. The sampler was located $21 \mathrm{~m}$ above the ground level, in the city centre (532 $\left.26^{\prime} 26^{\prime \prime} \mathrm{N}, 14^{\circ} 32^{\prime} 50^{\prime \prime} \mathrm{E}\right)$. The sampler drums were changed weekly, and the tapes cut into $48-\mathrm{mm}$ segments representing the previous 7 days. Average daily concentrations were presented as the number of pollen grains per cubic meter per $24 \mathrm{~h}$.

Consecutive phases of the pollen season were distinguished as starting with 1, 5, 25, 50, 75 and 99\% of the seasonal sum of pollen of each taxon studied. The course of the pollen season (Figure 1-5) was presented in the way proposed by Latałowa et al. (2002). Duration of the pollen seasons of each taxon was defined as the period in which $98 \%$ of the total catch occurred (S pieksma et al. 1995, Jato et al. 2006). The main pollen season was established as proposed by Nils s on and Pers s on (1981) as the period from which the sum of daily mean concentrations reached $5 \%$ of the total sum until the time when the sum achieved $95 \%$ of the total sum. On the basis of literature data, the number of days on which the pollen count of the taxa studied exceeded the threshold values at which the first allergy symptoms develop, was determined. Rapie jk o et al. (2004) reported a threshold value for Artemisia pollen of 30 grains $\times \mathrm{m}^{-3}$, R odrigue z - R a jo et al. (2003) announced an allergenic significant value for Urticaceae pollen of 80 grains $\times \mathrm{m}^{-3}$, for Plantago, Rumex and Chenopodiaceae pollen of 50 grains $\times \mathrm{m}^{-3}$.

The meteorological data were provided by the Automatic Weather Station (Vaisala, Finland), located at the monitoring site. The meteorological parameters taken into consideration in assessment of the effect of meteorological conditions on airborne pollen were as follows: daily precipitation total, wind speed, relative humidity, air temperature and dew point. The degree of correlation between particular meteorological parameters and the concentration of grass pollen was described by the multiple regression model and Pearson's correlations (a statistical error risk was estimated at the significance level of $95 \%, \alpha=0.05$ ) using the Statistica version 0.9 (StatSoft. Inc., 2008).

\section{RESULTS}

Rumex: The diagrams presenting the dynamics of the Rumex pollen seasons are slightly asymmetric. In 2005 and 2006 they are skewed to the right, the final phases were insignificantly longer than the initial ones (Fig. 1A). The initial and terminal stages (5\% and $95 \%$ ) were very long in 2004 and 2005, with the main middle phases being short, when $50 \%$ (25-75\%) of the total pollen count occurred in the air. The longest main stage was noted in 2008, accompanied by the shortest other phases. The pollen season was the longest in 2004 and the shortest in 2006; in both cases it was calculated using two methods $-98 \%$ and $90 \%$. Despite the long pollen seasons of sorrel (about 2-3 months), the risk from pollen allergens of this taxon in the period studied was very low or nonexistent (Tab. 1 ). The dates of maximum values of pollen count were recorded before the value of $50 \%$ of total pollen sum was reached in successive years, except the year 2004. In that year (2004), the maximum pollen concentration was noted 12 days after $50 \%$ of total pollen sum was reached (Fig. 1B, Tab. 1).

The most important agents influencing the Rumex pollen count (in multiple regression) are wind speed, air humidity and maximum temperature (Table 2).

Plantago: The dynamics of the plantain pollen season was similar in each of the 5 years of the study period. The differences in duration of each particular stage (1-5\%, 5-25\%, 25-50\%, 50-75\%, 75-95\%, 95-99\%) were not longer than a few days (Fig. 2A). The pollen seasons of the Plantago genus were long and showed many peaks in successive years (Fig. 2B). The maximum pollen counts were observed on different dates from the $16^{\text {th }}$ of July to the $10^{\text {th }}$ of August after the value of $50 \%$ of total pollen sum was reached, except the years 2005 and 2007. In these years, the date of maximum value was noted exactly on the day when a half of total pollen sum was recorded. However, in 2006 the maximum pollen counts occurred in the final stage (75-95\%) (Fig. 1A, 2B). The longest terminal phases (75-95\%) were noted in 2007 and 2008. During the 5 years of this study, the Plantago pollen count did not exceed the threshold value, so no risk from pollen allergens of this taxon was noted in Szczecin in this period (Tab. 1).

In multiple regression analysis, significant correlations between the pollen count and wind speed and air temperature were noted only in 2006, however, with respect to air humidity and dew point only in 2007 . In 2005, 2007 and 2008, the most important weather factors in Pearson's correlation were humidity and maximum air temperature (Tab. 2).

Chenopodiaceae: In the 5 years of study, the diagrams describing the pollen seasons of Chenopodiaceae were irregular. The longest initial stage (5-25\%) occurred in 2007, while the longest final stage (75-95\%) in 2004 (Fig. 3A). The earliest dates of the inceptive stage (1-5\%) were recorded in 2007 and 2008 (first grains appeared already in the end of May). As the family Chenopodiaceae is represented by many taxa, the pollen seasons were long and the diagrams obtained in successive years had many peaks noted on different dates from the $27^{\text {th }}$ of July to the $19^{\text {th }}$ of August. The dates of maximum pollen count occurred in the middle phases 
Table. 1

Characteristic of the main pollen seasons - MPS ( $90 \%$ of total pollen sum) and number of days with pollen count over the allergenically significant value.

\begin{tabular}{|c|c|c|c|c|c|c|}
\hline Taxon & & 2004 & 2005 & 2006 & 2007 & 2008 \\
\hline \multirow[t]{5}{*}{ Rumex } & $M P S$ & $15 \mathrm{~V}-12$ IX & $30 \mathrm{~V}-29 \mathrm{VIII}$ & 2 VI-9 VIII & 22 V-9 VIII & $21 \mathrm{~V}-7 \mathrm{VIII}$ \\
\hline & Length & 109 & 91 & 69 & 83 & 82 \\
\hline & Max/date & 28/8 VIII & $40 / 24 \mathrm{VI}$ & $38 / 14 \mathrm{VI}$ & $50 / 6 \mathrm{VI}$ & 30/4 VI \\
\hline & Total pollen & 819 & 842 & 836 & 906 & 730 \\
\hline & Days $>50 p / m^{3}$ & 0 & 0 & 0 & 1 & 0 \\
\hline \multirow[t]{5}{*}{ Plantago } & $M P S$ & 6 VI-12 IX & 11 VI-29 VIII & 15 VI-22 VIII & 6 VI-5 IX & $26 \mathrm{~V}-31 \mathrm{VIII}$ \\
\hline & Length & 97 & 80 & 69 & 92 & 98 \\
\hline & Max/date & 6/6 VIII & $12 / 10 \mathrm{VII}$ & 13/7 VIII & 13/16 VII & $14 / 20 \mathrm{VII}$ \\
\hline & Total pollen & 204 & 233 & 190 & 155 & 200 \\
\hline & Days $>50 p / m^{3}$ & 0 & 0 & 0 & 0 & 0 \\
\hline \multirow[t]{5}{*}{ Chenopodiaceae } & $M P S$ & 11 VII-2 X & 4 VII-10 IX & 1 VII-7 IX & 27 VI-30 VIII & 8 VII-9 IX \\
\hline & Length & 84 & 68 & 69 & 66 & 64 \\
\hline & Max/date & 21/7 VIII & 7/19 VIII & 10/28 VII & 17/14 VIII & 7/27 VII \\
\hline & Total pollen & 229 & 145 & 134 & 122 & 114 \\
\hline & Days $>50 \mathrm{p} / \mathrm{m}^{3}$ & 0 & 0 & 0 & 0 & 0 \\
\hline \multirow[t]{5}{*}{ Artemisia } & $M P S$ & 28 VII-23VIII & 25 VII-30VIII & 13 VII-3IX & 25 VII-20VIII & 24 VII-30VIII \\
\hline & Length & 27 & 37 & 53 & 27 & 38 \\
\hline & Max/date & 182/9 VIII & $60 / 15$ VIII & 30/19 VIII & 99/6 VIII & 33/27 VII \\
\hline & Total pollen & 1598 & 706 & 385 & 817 & 390 \\
\hline & Days $>30 p / m^{3}$ & 14 & 7 & 1 & 9 & 2 \\
\hline \multirow[t]{5}{*}{ Urticaceae } & $M P S$ & 26 VI-29 VIII & 25 VI-29 VIII & $30 \mathrm{VI}-31 \mathrm{VIII}$ & 5 VII-24 VIII & 27 VI-5 IX \\
\hline & Length & 65 & 66 & 63 & 51 & 71 \\
\hline & Max/date & $573 / 23$ VII & $306 / 28$ VII & 288/19 VIII & 1123/13 VIII & 398/27 VII \\
\hline & Total pollen & 15166 & 6612 & 8505 & 18212 & 6392 \\
\hline & Days $>80 p / m^{3}$ & 59 & 36 & 44 & 55 & 24 \\
\hline
\end{tabular}

(25-75\%) in the whole period of study (Fig. 3B, Tab. 1). Similarly as for the plantain pollen, the Chenopodiaceae pollen concentration did not exceed the threshold value at which allergy symptoms can appear.

Significant correlations between the pollen count of this taxon and humidity as well as temperature were found only in 2006 and 2008 (Tab. 2).

Artemisia: The diagrams for compact pollen seasons of mugwort are asymmetric. The earliest dates of the initial phases (1-5\%) were noted in 2006 and 2008 , and the latest in 2004. The final stages $(95-99 \%)$ lasted the longest in 2004 and 2008. The main phases $(25-75 \%)$ were very short in 2004 and 2007 and lasted only a few days (Fig. 4A). The maximum values of pollen count were observed in a short span (23 days) from the $27^{\text {th }}$ of July to the $19^{\text {th }}$ of August. For most periods studied, the day of the highest concentration occurred approximately in the middle of the season, except the year 2008. In that year, the date of maximum value was noted in the initial phase (5-25\%) (Fig. 4A, 4B). The Artemisia pollen count exceeded the threshold value in Szczecin for a period of 1 to 14 days in 2006 and 2004, respectively (Tab. 1).
The main weather factors influencing the pollen count of mugwort are air humidity, maximum temperature and dew point (Tab. 2).

Urtica: The dynamics of the nettle pollen season was similar in the 5 years studied; the durations of particular stages were also similar (Fig. 5A). As the family Urticaceae is represented by many taxa, the pollen seasons were very long and lasted in Szczecin from 51 days in 2007 to 71 days in 2008 (Fig. 5B). The maximum pollen concentrations were noted over a period of one month, from the $23^{\text {rd }}$ of July to the $19^{\text {th }}$ of August. The longest final stages (75-95\%) were noted in 2007 and 2008. The Urtica pollen count exceeded the threshold value in Szczecin for a period of 51 to 71 days, over which allergy symptoms could appear (Tab. 1).

The multiple regression showed strong correlations with rain and wind speed in 2004 and 2008, while in 2004, 2005 and 2006 a correlation was noted with the dew point. Pearson's analysis revealed significant correlations of the pollen count with air humidity, temperature and dew point (Tab. 2). 
Table 2

Pollen counts for selected herbaceous taxa vs. meteorological parameters in the multiple regression model and Pearson's coefficient, in Szczecin (2004-2008)

\begin{tabular}{|c|c|c|c|c|c|c|c|c|}
\hline Taxon & Year & Period/N & $\begin{array}{c}\text { Variance analysis at } \\
\alpha \alpha=0.05\end{array}$ & $\begin{array}{l}\text { Adjusted } \\
\text { R-square }\end{array}$ & $\begin{array}{c}\text { Explanatory } \\
\text { variables }\end{array}$ & $\begin{array}{l}\text { Explained } \\
\text { variation } \\
\text { (R-square) }\end{array}$ & $\begin{array}{l}\text { Partial } \\
\text { correl. }\end{array}$ & $\begin{array}{c}\text { Pearson's } \\
\text { coefficient } \\
* \text {-statistic } \\
\text { significant } \\
\alpha<0.05\end{array}$ \\
\hline \multirow{5}{*}{ Rumex } & 2004 & $\begin{array}{l}15.05- \\
-12.09 \\
N=136\end{array}$ & $\begin{array}{l}F=15.89537 \\
P=0.000000\end{array}$ & 0.4357 & $\begin{array}{l}\text { Wind }_{\text {max }} \\
\text { Rain } \\
\text { Humidity } \\
\mathbf{T}_{\text {max }} \\
\text { Dew point }\end{array}$ & $\begin{array}{c}-0.030 \\
-0.350 \\
0.021 \\
\mathbf{0 . 2 7 4} \\
0.160 \\
\end{array}$ & $\begin{array}{l}- \\
- \\
- \\
+ \\
-\end{array}$ & $\begin{array}{c}-0.08 \\
-\mathbf{0 . 4 0 *} \\
0.02 \\
\mathbf{0 . 6 5} * \\
\mathbf{0 . 4 0} * \\
\end{array}$ \\
\hline & 2005 & $\begin{array}{l}30.05- \\
-29.08 \\
N=116\end{array}$ & $\begin{array}{l}F=5.56745 \\
P=0.00005\end{array}$ & 0.1907 & $\begin{array}{l}\text { Wind }_{\max } \\
\text { Rain } \\
\text { Humidity } \\
\mathrm{T}_{\max } \\
\text { Dew point }\end{array}$ & $\begin{array}{c}0.057 \\
-0.030 \\
\mathbf{- 1 . 7 0 0} \\
-0.040 \\
1.210 \\
\end{array}$ & $\begin{array}{l}- \\
- \\
+ \\
- \\
-\end{array}$ & $\begin{array}{c}0.03 \\
-0.09 \\
\mathbf{- 0 . 3 6}^{*} \\
\mathbf{0 . 3 8} * \\
\mathbf{0 . 1 8} *\end{array}$ \\
\hline & 2006 & $\begin{array}{l}02.06- \\
-09.08 \\
\mathrm{~N}=97\end{array}$ & $\begin{array}{l}F=3.112781 \\
P=0.018030\end{array}$ & 0.0679 & $\begin{array}{l}\text { Wind }_{\text {max }} \\
\text { Rain } \\
\text { Humidity } \\
\mathbf{T}_{\text {max }} \\
\text { Dew point }\end{array}$ & $\begin{array}{c}\mathbf{0 . 2 3 0} \\
-0.100 \\
-0.070 \\
\mathbf{1 . 3 1 8} \\
-0.060\end{array}$ & $\begin{array}{l}+ \\
- \\
- \\
+ \\
-\end{array}$ & $\begin{array}{c}\mathbf{0 . 2 7} * \\
\mathbf{- 0 . 2 4} * \\
-0.19 \\
\mathbf{0 . 3 4} * \\
\mathbf{0 . 3 4} *\end{array}$ \\
\hline & 2007 & $\begin{array}{l}22.05- \\
-09.08 \\
N=104\end{array}$ & $\begin{array}{l}F=6.927970 \\
P=0.000004\end{array}$ & 0.2567 & $\begin{array}{l}\text { Wind }_{\text {max }} \\
\text { Rain } \\
\text { Humidity } \\
\mathrm{T}_{\max } \\
\text { Dew point }\end{array}$ & $\begin{array}{c}-0.040 \\
0.042 \\
0.238 \\
0.022 \\
-0.850 \\
\end{array}$ & $\begin{array}{l}- \\
- \\
- \\
- \\
-\end{array}$ & $\begin{array}{c}-0.10 \\
-0.03 \\
-\mathbf{0 . 4 6} \\
\mathbf{0 . 4 9 *} \\
\mathbf{0 . 2 5 *}\end{array}$ \\
\hline & 2008 & $\begin{array}{l}21.05- \\
-07.08 \\
N=106\end{array}$ & $\begin{array}{l}F=0.531610 \\
P=0.000000\end{array}$ & 0.4171 & $\begin{array}{l}\text { Wind }_{\text {max }} \\
\text { Rain } \\
\text { Humidity } \\
\text { T }_{\text {mid }} \\
\text { Dew point }\end{array}$ & $\begin{array}{c}-0.030 \\
-0.004 \\
0.549 \\
\mathbf{1 . 2 9 0} \\
-1.100 \\
\end{array}$ & $\begin{array}{l}- \\
- \\
- \\
+ \\
-\end{array}$ & $\begin{array}{c}0.11 \\
\mathbf{- 0 . 2 1} * \\
\mathbf{- 0 . 6 4} * \\
0.44^{*} \\
\mathbf{- 0 . 2 9}^{*}\end{array}$ \\
\hline \multirow{5}{*}{ Plantago } & 2004 & $\begin{array}{l}06.06- \\
-12.09 \\
N=97\end{array}$ & $\begin{array}{l}F=0.753760 \\
P=0.627300\end{array}$ & -0.0182 & $\begin{array}{l}\text { Wind }_{\text {max }} \\
\text { Rain } \\
\text { Humidity } \\
\mathrm{T}_{\text {max }} \\
\text { Dew point }\end{array}$ & $\begin{array}{c}0.070 \\
0.032 \\
-0.150 \\
0.128 \\
-0.060 \\
\end{array}$ & $\begin{array}{l}- \\
- \\
- \\
- \\
-\end{array}$ & $\begin{array}{c}-0.08 \\
-0.12 \\
-0.14 \\
0.17 \\
0.08\end{array}$ \\
\hline & 2005 & $\begin{array}{l}11.06- \\
-29.08 \\
N=87\end{array}$ & $\begin{array}{l}F=2.769469 \\
P=0.016922\end{array}$ & 0.1098 & $\begin{array}{l}\text { Wind }_{\text {max }} \\
\text { Rain } \\
\text { Humidity } \\
\mathrm{T}_{\text {max }} \\
\text { Dew point }\end{array}$ & $\begin{array}{c}-0.020 \\
0.123 \\
-0.560 \\
0.153 \\
0.483 \\
\end{array}$ & $\begin{array}{l}- \\
- \\
- \\
- \\
-\end{array}$ & $\begin{array}{c}-0.05 \\
0.06 \\
\mathbf{- 0 . 3 3}^{*} \\
\mathbf{0 . 3 6}^{*} \\
0.14 \\
\end{array}$ \\
\hline & 2006 & $\begin{array}{l}15.06- \\
-22.08 \\
N=69\end{array}$ & $\begin{array}{l}F=2.556622 \\
P=0.028716\end{array}$ & 0.1256 & $\begin{array}{l}\text { Wind }_{\max } \\
\text { Rain } \\
\text { Humidity } \\
\mathbf{T}_{\text {max }} \\
\text { Dew point }\end{array}$ & $\begin{array}{c}\mathbf{- 0 . 3 1 0} \\
-0.020 \\
-0.120 \\
\mathbf{0 . 7 7 9} \\
-0.470 \\
\end{array}$ & $\begin{array}{l}+ \\
- \\
- \\
+ \\
-\end{array}$ & $\begin{array}{c}\mathbf{- 0 . 3 2} * \\
-0.03 \\
-0.10 \\
0.23 \\
0.16 \\
\end{array}$ \\
\hline & 2007 & $\begin{array}{l}06.06- \\
-05.09 \\
N=92\end{array}$ & $\begin{array}{l}F=5.922833 \\
P=0.000056\end{array}$ & 0.2937 & $\begin{array}{l}\text { Wind }_{\text {max }} \\
\text { Rain } \\
\text { Humidity } \\
\mathrm{T}_{\text {max }} \\
\text { Dew point }\end{array}$ & $\begin{array}{c}0.174 \\
-0.060 \\
\mathbf{1 . 9 9 0} \\
0.511 \\
\mathbf{- 2 . 9 0 0} \\
\end{array}$ & $\begin{array}{l}- \\
- \\
+ \\
- \\
+\end{array}$ & $\begin{array}{c}0.04 \\
-0.05 \\
-\mathbf{0 . 3 8} * \\
\mathbf{0 . 5 3} * \\
\mathbf{0 . 3 9} * \\
\end{array}$ \\
\hline & 2008 & $\begin{array}{l}26.05- \\
-31.08 \\
N=98\end{array}$ & $\begin{array}{l}F=2.180965 \\
P=0.054975\end{array}$ & 0.0852 & $\begin{array}{l}\text { Wind }_{\text {max }} \\
\text { Rain } \\
\text { Humidity } \\
\mathrm{T}_{\text {max }} \\
\text { Dew point }\end{array}$ & $\begin{array}{c}0.050 \\
0.122 \\
-0.150 \\
0.691 \\
0.175\end{array}$ & $\begin{array}{l}- \\
- \\
- \\
- \\
-\end{array}$ & $\begin{array}{c}0.04 \\
0.08 \\
-0.19 \\
\mathbf{0 . 3 6 *} \\
0.11\end{array}$ \\
\hline
\end{tabular}


cd. Table 2

\begin{tabular}{|c|c|c|c|c|c|c|c|c|}
\hline Taxon & Year & Period/N & $\begin{array}{c}\text { Variance analysis at } \\
\qquad \alpha=0.05\end{array}$ & $\begin{array}{l}\text { Adjusted } \\
\text { R-square }\end{array}$ & $\begin{array}{l}\text { Explanatory } \\
\text { variables }\end{array}$ & $\begin{array}{l}\text { Explained } \\
\text { variation } \\
\text { (R-square) }\end{array}$ & $\begin{array}{l}\text { Partial } \\
\text { correl. }\end{array}$ & $\begin{array}{c}\text { Pearson's } \\
\text { coefficient } \\
*_{\text {-statistic }} \\
\text { significant } \\
\alpha<0.05\end{array}$ \\
\hline \multirow{5}{*}{ Chenopodiaceae } & 2004 & $\begin{array}{l}11.07- \\
-02.10 \\
N=84\end{array}$ & $\begin{array}{l}F=6.858655 \\
P=0.000004\end{array}$ & 0.3535 & $\begin{array}{l}\text { Wind }_{\text {max }} \\
\text { Rain } \\
\text { Humidity } \\
\mathbf{T}_{\text {max }} \\
\text { Dew point }\end{array}$ & $\begin{array}{c}0.005 \\
-0.340 \\
-0.060 \\
\mathbf{1 . 1 6 1} \\
-0.080 \\
\end{array}$ & $\begin{array}{l}- \\
- \\
- \\
+ \\
-\end{array}$ & $\begin{array}{c}0.03 \\
\mathbf{- 0 . 5 2} * \\
-0.08 \\
\mathbf{0 . 5 4} * \\
0.20 \\
\end{array}$ \\
\hline & 2005 & $\begin{array}{l}04.07- \\
-10.10 \\
N=68\end{array}$ & $\begin{array}{l}F=0.893253 \\
P=0.505873\end{array}$ & -0.0099 & $\begin{array}{l}\text { Wind }_{\text {max }} \\
\text { Rain } \\
\text { Humidity } \\
\mathrm{T}_{\text {max }} \\
\text { Dew point }\end{array}$ & $\begin{array}{c}-0.020 \\
-0.140 \\
-0.450 \\
0.383 \\
0.452 \\
\end{array}$ & $\begin{array}{l}- \\
- \\
- \\
- \\
-\end{array}$ & $\begin{array}{c}-0.08 \\
-0.20 \\
-0.12 \\
0.21 \\
0.04 \\
\end{array}$ \\
\hline & 2006 & $\begin{array}{l}01.07- \\
-07.09 \\
N=69\end{array}$ & $\begin{array}{l}F=1.166415 \\
P=0.337063\end{array}$ & 0.0156 & $\begin{array}{l}\text { Wind }_{\text {max }} \\
\text { Rain } \\
\text { Humidity } \\
\mathrm{T}_{\text {max }} \\
\text { Dew point }\end{array}$ & $\begin{array}{c}0.113 \\
-0.010 \\
\mathbf{0 . 5 4 2} \\
-0.050 \\
-0.490 \\
\end{array}$ & $\begin{array}{l}- \\
- \\
+ \\
- \\
-\end{array}$ & $\begin{array}{c}0.07 \\
-0.07 \\
\mathbf{- 0 . 2 9 *} \\
\mathbf{0 . 2 5} * \\
-0.10 \\
\end{array}$ \\
\hline & 2007 & $\begin{array}{l}27.06- \\
-30.08 \\
N=66\end{array}$ & $\begin{array}{l}F=1,303092 \\
P=0.274430\end{array}$ & 0.0331 & $\begin{array}{l}\text { Wind }_{\text {max }} \\
\text { Rain } \\
\text { Humidity } \\
\mathrm{T}_{\max } \\
\text { Dew point }\end{array}$ & $\begin{array}{c}0.039 \\
-0.070 \\
-2.200 \\
-0.410 \\
3.470 \\
\end{array}$ & $\begin{array}{l}- \\
- \\
- \\
- \\
-\end{array}$ & $\begin{array}{c}0.04 \\
-0.12 \\
-0.15 \\
\mathbf{0 . 2 7} * \\
0.23 \\
\end{array}$ \\
\hline & 2008 & $\begin{array}{l}08.07- \\
-09.09 \\
N=64\end{array}$ & $\begin{array}{l}F=4.923386 \\
P=0.000681\end{array}$ & 0.3290 & $\begin{array}{l}\text { Wind }_{\text {max }} \\
\text { Rain } \\
\text { Humidity } \\
\mathbf{T}_{\text {max }} \\
\text { Dew point }\end{array}$ & $\begin{array}{c}0.008 \\
0.028 \\
\mathbf{- 2 . 2 0 0} \\
\mathbf{2 . 0 8 5} \\
1.620 \\
\end{array}$ & $\begin{array}{l}- \\
- \\
+ \\
+ \\
-\end{array}$ & $\begin{array}{c}0.15 \\
-0.11 \\
\mathbf{- 0 . 4 7}^{*} \\
\mathbf{0 . 5 8} * \\
0.19 \\
\end{array}$ \\
\hline \multirow{5}{*}{ Artemisia } & 2004 & $\begin{array}{l}28.07- \\
-23.08 \\
N=27\end{array}$ & $\begin{array}{l}F=14.02587 \\
P=0.000000\end{array}$ & 0.5520 & $\begin{array}{l}\text { Wind }_{\text {max }} \\
\text { Rain } \\
\text { Humidity } \\
\mathbf{T}_{\text {max }} \\
\text { Dew point }\end{array}$ & $\begin{array}{c}-0.030 \\
-0.370 \\
-0.080 \\
\mathbf{1 . 3 1 7} \\
0.017\end{array}$ & $\begin{array}{l}- \\
- \\
- \\
+ \\
-\end{array}$ & $\begin{array}{c}-0.09 \\
-\mathbf{0 . 5 8} * \\
-0.14 \\
\mathbf{0 . 7 0} * \\
\mathbf{0 . 3 0} *\end{array}$ \\
\hline & 2005 & $\begin{array}{l}25.07- \\
-30.08 \\
N=37\end{array}$ & $\begin{array}{l}F=1.345734 \\
P=0.249775\end{array}$ & 0.0280 & $\begin{array}{l}\text { Wind }_{\text {max }} \\
\text { Rain } \\
\text { Humidity } \\
\mathbf{T}_{\text {max }} \\
\text { Dew point }\end{array}$ & $\begin{array}{c}0.007 \\
0.022 \\
\mathbf{- 3 . 4 0 0} \\
\mathbf{- 0 . 8 1 0} \\
\mathbf{3 . 5 7 0} \\
\end{array}$ & $\begin{array}{l}- \\
- \\
+ \\
+ \\
+\end{array}$ & $\begin{array}{c}0.00 \\
0.10 \\
0.06 \\
-0.15 \\
-0.03 \\
\end{array}$ \\
\hline & 2006 & $\begin{array}{l}13.07- \\
-03.09 \\
N=53\end{array}$ & $\begin{array}{l}F=0.475437 \\
P=0.823893\end{array}$ & 0.5088 & $\begin{array}{l}\text { Wind }_{\text {max }} \\
\text { Rain } \\
\text { Humidity } \\
\mathrm{T}_{\text {max }} \\
\text { Dew point }\end{array}$ & $\begin{array}{c}-0.200 \\
0.036 \\
-0.120 \\
-0.130 \\
0.142\end{array}$ & $\begin{array}{l}- \\
- \\
- \\
-\end{array}$ & $\begin{array}{c}-0.19 \\
0.01 \\
0.00 \\
0.05 \\
0.09\end{array}$ \\
\hline & 2007 & $\begin{array}{l}25.07- \\
-20.08 \\
N=27\end{array}$ & $\begin{array}{l}F=1.930694 \\
P=0.096929\end{array}$ & 0.1004 & $\begin{array}{l}\text { Wind }_{\text {max }} \\
\text { Rain } \\
\text { Humidity } \\
\mathrm{T}_{\text {max }} \\
\text { Dew point }\end{array}$ & $\begin{array}{c}0.169 \\
-0.087 \\
\mathbf{- 2 . 6 0 0} \\
\mathbf{- 1 . 0 9 0} \\
3.960\end{array}$ & $\begin{array}{l}- \\
- \\
+ \\
+ \\
-\end{array}$ & $\begin{array}{c}0.16 \\
-0.08 \\
\mathbf{- 0 . 3 3} * \\
\mathbf{0 . 2 9} * \\
0.13\end{array}$ \\
\hline & 2008 & $\begin{array}{l}24.07- \\
-30.08 \\
N=38\end{array}$ & $\begin{array}{l}F=10.84925 \\
P=0.000000\end{array}$ & 0.4962 & $\begin{array}{l}\text { Wind }_{\text {max }} \\
\text { Rain } \\
\text { Humidity } \\
\mathbf{T}_{\text {mid }} \\
\text { Dew point }\end{array}$ & $\begin{array}{c}-0.040 \\
-0.060 \\
1.800 \\
\mathbf{2 . 9 2 0} \\
\mathbf{- 1 . 9 0 0}\end{array}$ & $\begin{array}{l}- \\
- \\
- \\
+ \\
+\end{array}$ & $\begin{array}{c}0.08 \\
-0.19 \\
-\mathbf{- 0 . 5 5} \\
\mathbf{0 . 7 0} \\
\mathbf{0 . 2 8}^{*}\end{array}$ \\
\hline
\end{tabular}


cd. Table 2

\begin{tabular}{|c|c|c|c|c|c|c|c|c|}
\hline Taxon & Year & Period/N & $\begin{array}{c}\text { Variance analysis at } \\
\qquad \alpha=0.05\end{array}$ & $\begin{array}{l}\text { Adjusted } \\
\text { R-square }\end{array}$ & $\begin{array}{c}\text { Explanatory } \\
\text { variables }\end{array}$ & $\begin{array}{l}\text { Explained } \\
\text { variation } \\
\text { (R-square) }\end{array}$ & $\begin{array}{l}\text { Partial } \\
\text { correl. }\end{array}$ & $\begin{array}{c}\text { Pearson's } \\
\text { coefficient } \\
\text { *-statistic }_{\text {sticant }} \\
\text { significa } \\
\alpha<0.05\end{array}$ \\
\hline \multirow{5}{*}{ Urtica } & 2004 & $\begin{array}{l}26.06- \\
-29.08 \\
N=65\end{array}$ & $\begin{array}{l}F=23.57482 \\
P=0.000000\end{array}$ & 0.5725 & $\begin{array}{l}\text { Wind }_{\text {max }} \\
\text { Rain } \\
\text { Humidity } \\
\mathrm{T}_{\text {max }} \\
\text { Dew point }\end{array}$ & $\begin{array}{c}\mathbf{- 0 . 1 5 0} \\
-\mathbf{0 . 5 4 0} \\
0.000 \\
-0.060 \\
\mathbf{0 . 7 2 4}\end{array}$ & $\begin{array}{l}+ \\
+ \\
- \\
- \\
+\end{array}$ & $\begin{array}{c}\mathbf{- 0 . 2 0 *} \\
\mathbf{- 0 . 3 2} * \\
0.04 \\
0.70^{*} \\
\mathbf{0 . 6 0} *\end{array}$ \\
\hline & 2005 & $\begin{array}{l}25.06- \\
-29.08 \\
N=66\end{array}$ & $\begin{array}{l}F=4.480331 \\
P=0.000465\end{array}$ & 0.1645 & $\begin{array}{l}\text { Wind }_{\text {max }} \\
\text { Rain } \\
\text { Humidity } \\
\mathrm{T}_{\text {max }} \\
\text { Dew point }\end{array}$ & $\begin{array}{l}-0.060 \\
-0.066 \\
-1.900 \\
-0.017 \\
\mathbf{2 . 6 2 0}\end{array}$ & $\begin{array}{l}- \\
- \\
- \\
- \\
+\end{array}$ & $\begin{array}{c}-0.09 \\
0.02 \\
-0.04 \\
\mathbf{0 . 3 1} * \\
\mathbf{0 . 4 1} *\end{array}$ \\
\hline & 2006 & $\begin{array}{l}30.08- \\
-31.08 \\
N=63\end{array}$ & $\begin{array}{l}F=6.401230 \\
P=0.000011\end{array}$ & 0.2411 & $\begin{array}{l}\text { Wind }_{\text {max }} \\
\text { Rain } \\
\text { Humidity } \\
\mathrm{T}_{\text {mid }} \\
\text { Dew point }\end{array}$ & $\begin{array}{c}-0.150 \\
0.017 \\
1.430 \\
\mathbf{2 . 2 4 0} \\
-0.990\end{array}$ & $\begin{array}{l}- \\
- \\
- \\
+ \\
-\end{array}$ & $\begin{array}{l}-0.15 \\
-0.04 \\
\mathbf{0 . 3 0} * \\
\mathbf{0 . 4 7} * \\
\mathbf{0 . 2 1} *\end{array}$ \\
\hline & 2007 & $\begin{array}{l}05.07- \\
-24.08 \\
N=51\end{array}$ & $\begin{array}{l}F=6.676010 \\
P=0.000004\end{array}$ & 0.2154 & $\begin{array}{l}\text { Wind }_{\text {max }} \\
\text { Rain } \\
\text { Humidity } \\
\mathrm{T}_{\text {max }} \\
\text { Dew point }\end{array}$ & $\begin{array}{c}0.049 \\
0.042 \\
-0.590 \\
-0.280 \\
0.746\end{array}$ & $\begin{array}{l}- \\
- \\
- \\
-\end{array}$ & $\begin{array}{c}-0.01 \\
0.01 \\
\mathbf{- 0 . 3 0 *} \\
\mathbf{0 . 4 5 *} \\
\mathbf{0 . 3 7} *\end{array}$ \\
\hline & 2008 & $\begin{array}{l}27.06- \\
-05.09 \\
N=71\end{array}$ & $\begin{array}{l}F=14.90953 \\
P=0.000000\end{array}$ & 0.4003 & $\begin{array}{l}\text { Wind }_{\text {max }} \\
\text { Rain } \\
\text { Humidity } \\
\mathrm{T}_{\max } \\
\text { Dew point }\end{array}$ & $\begin{array}{c}\mathbf{- 0 . 2 0 0} \\
-0.040 \\
-0.790 \\
0.032 \\
0.850\end{array}$ & $\begin{array}{l}+ \\
- \\
- \\
- \\
-\end{array}$ & $\begin{array}{c}-0.11 \\
-0.10 \\
\mathbf{- 0 . 2 6} * \\
\mathbf{0 . 5 4} * \\
\mathbf{0 . 4 4} *\end{array}$ \\
\hline
\end{tabular}



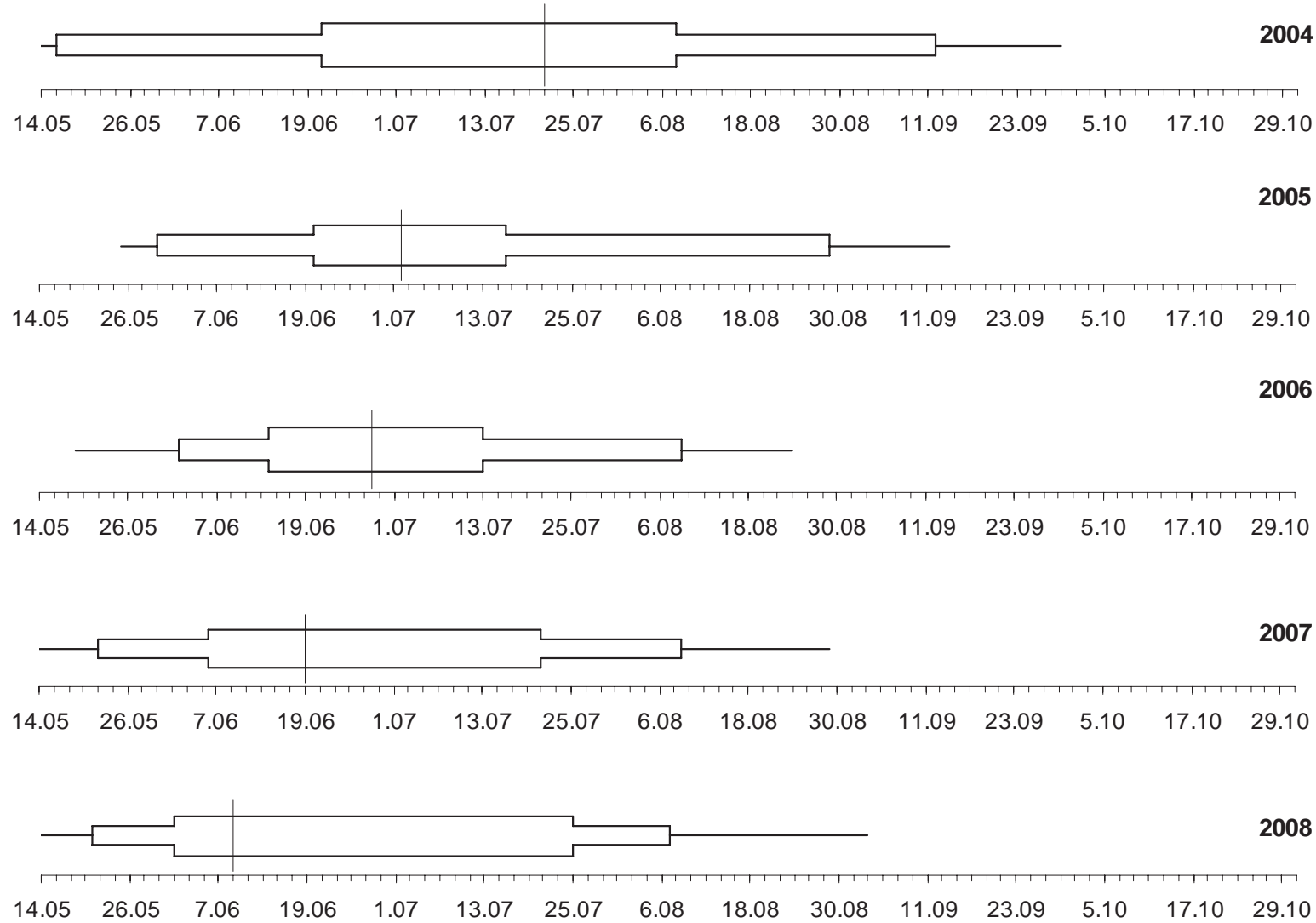

Fig. 1A. Dynamics of Rumex pollen seasons in Szczecin, 2004-2008. Diagrams of consecutive stages of the pollen season: 1\%, $5 \%, 25 \%, 50 \%$ (vertical line), 75\%, 95\%, $99 \%$.

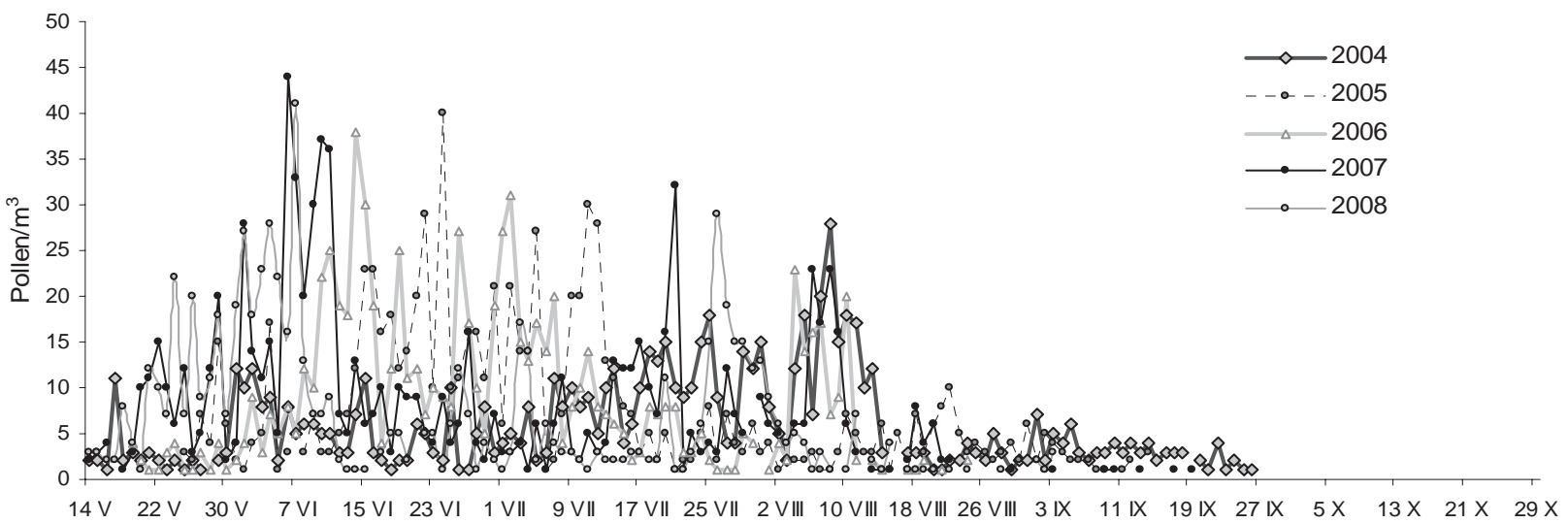

Fig. 1B. Seasonal values of Rumex pollen count in Szczecin, 2004-2008. 

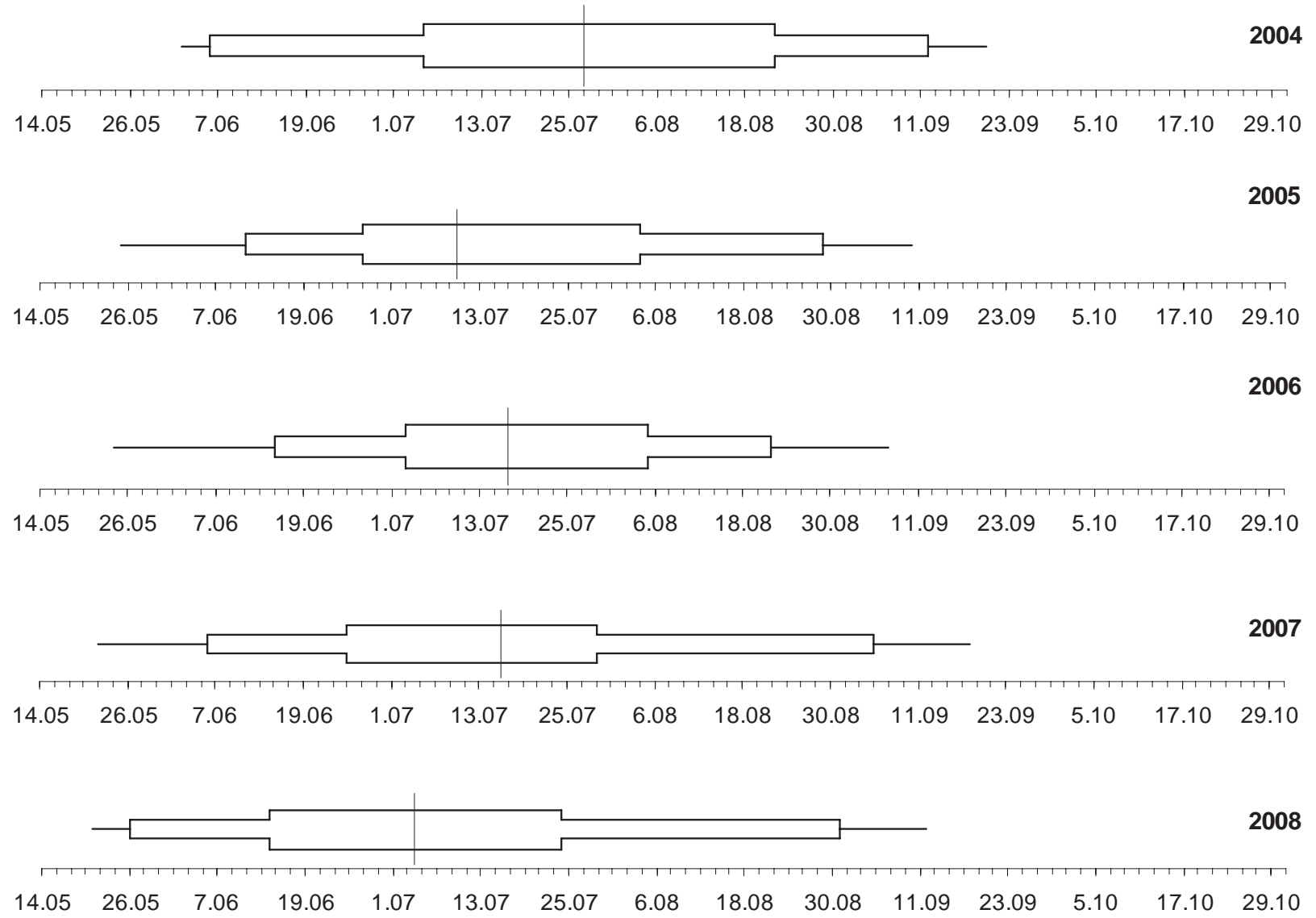

Fig. 2A. Dynamics of Plantago pollen seasons in Szczecin, 2004-2008. Diagrams of consecutive stages of the pollen season: 1\%, $5 \%, 25 \%, 50 \%$ (vertical line), $75 \%, 95 \%, 99 \%$.

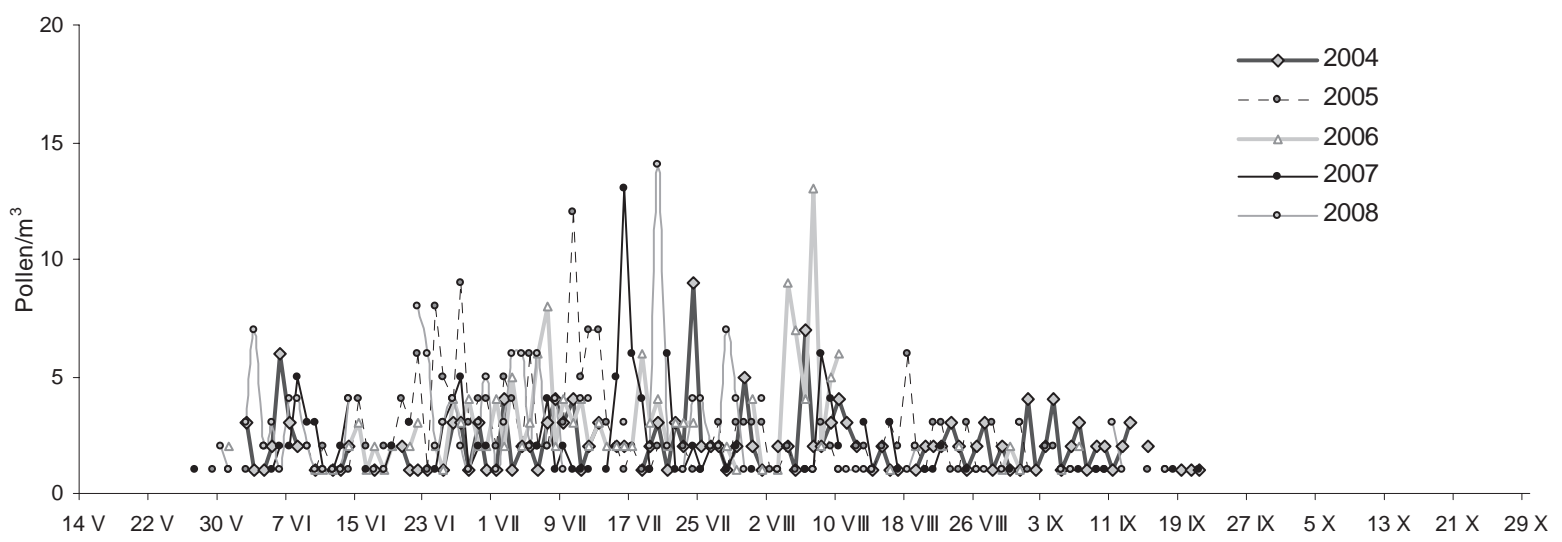

Fig. 2B. Seasonal values of Plantago pollen count in Szczecin, 2004-2008. 

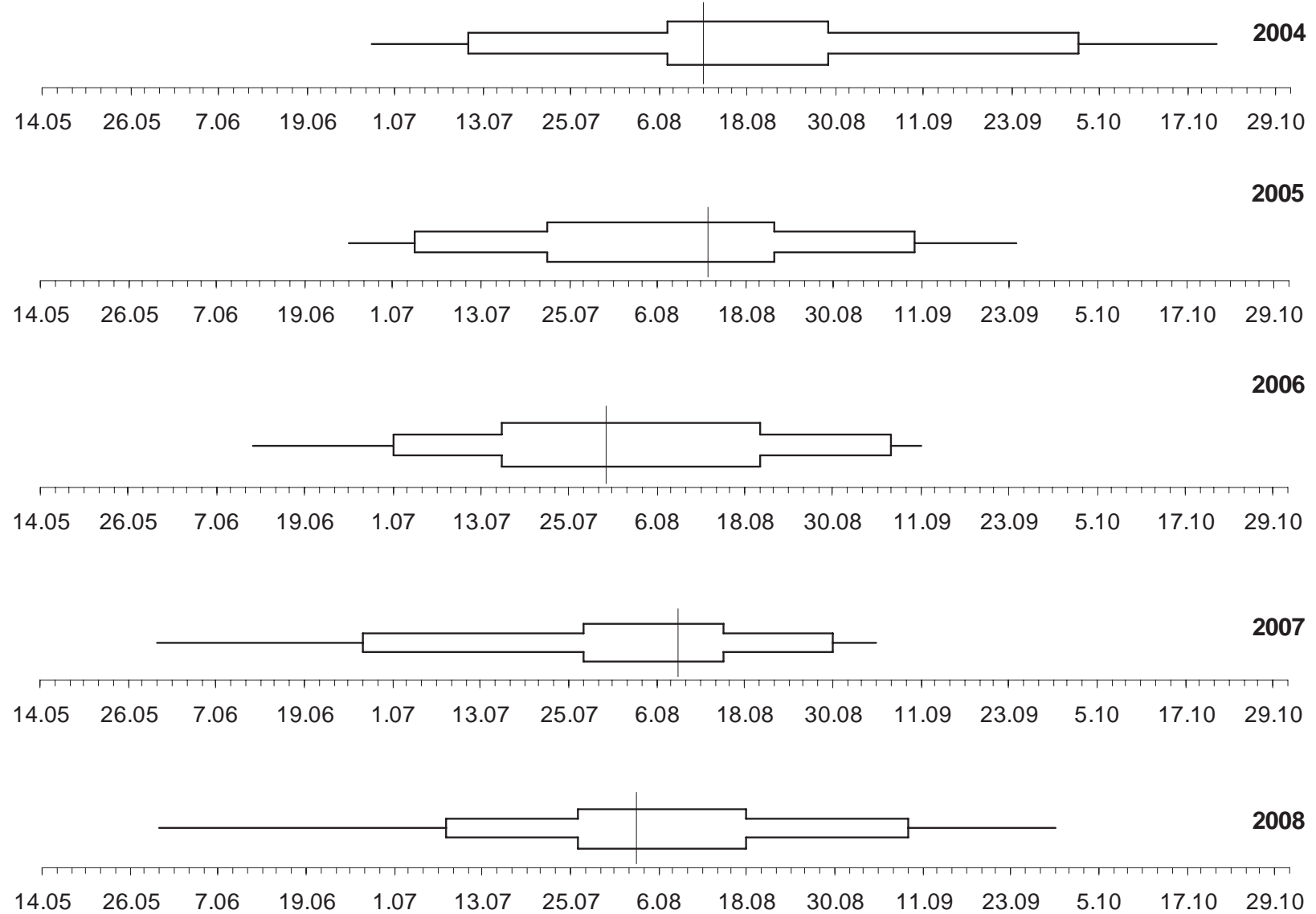

Fig. 3A. Dynamics of Chenopodiaceae pollen seasons in Szczecin, 2004-2008. Diagrams of consecutive stages of the pollen season: $1 \%, 5 \%, 25 \%, 50 \%$ (vertical line), $75 \%, 95 \%, 99 \%$.

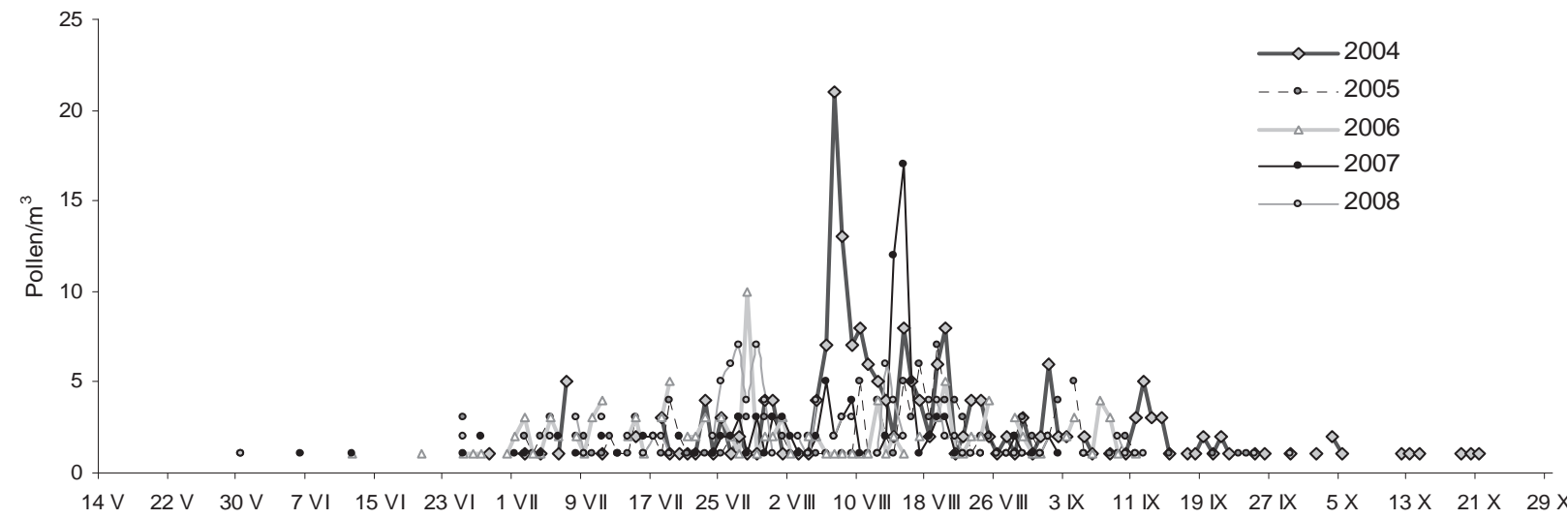

Fig. 3B. Seasonal values of Chenopodiaceae pollen count in Szczecin, 2004-2008. 

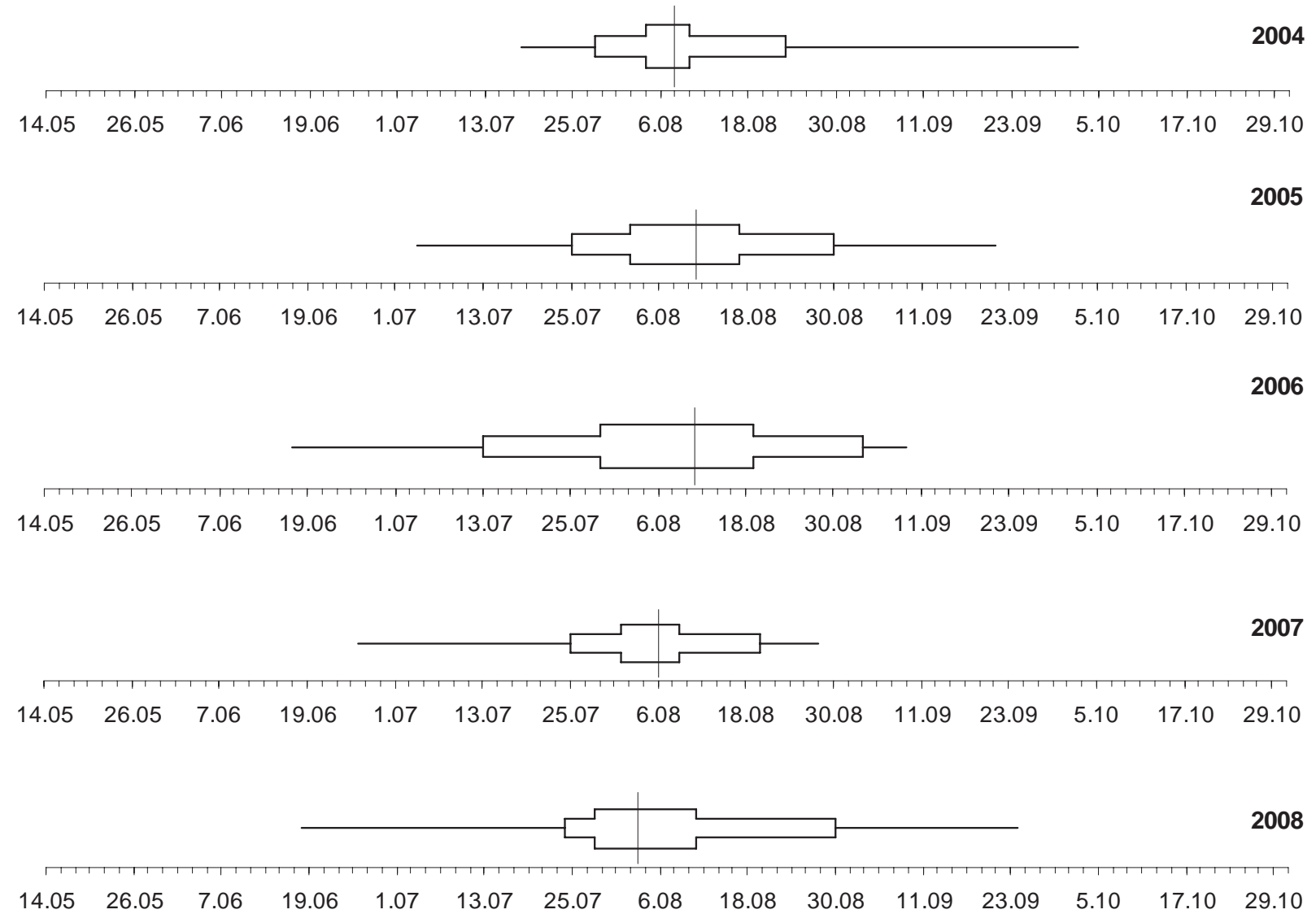

Fig. 4A. Dynamics of Artemisia pollen seasons in Szczecin, 2004-2008. Diagrams of consecutive stages of the pollen season: 1\%, $5 \%, 25 \%, 50 \%$ (vertical line), $75 \%, 95 \%, 99 \%$.

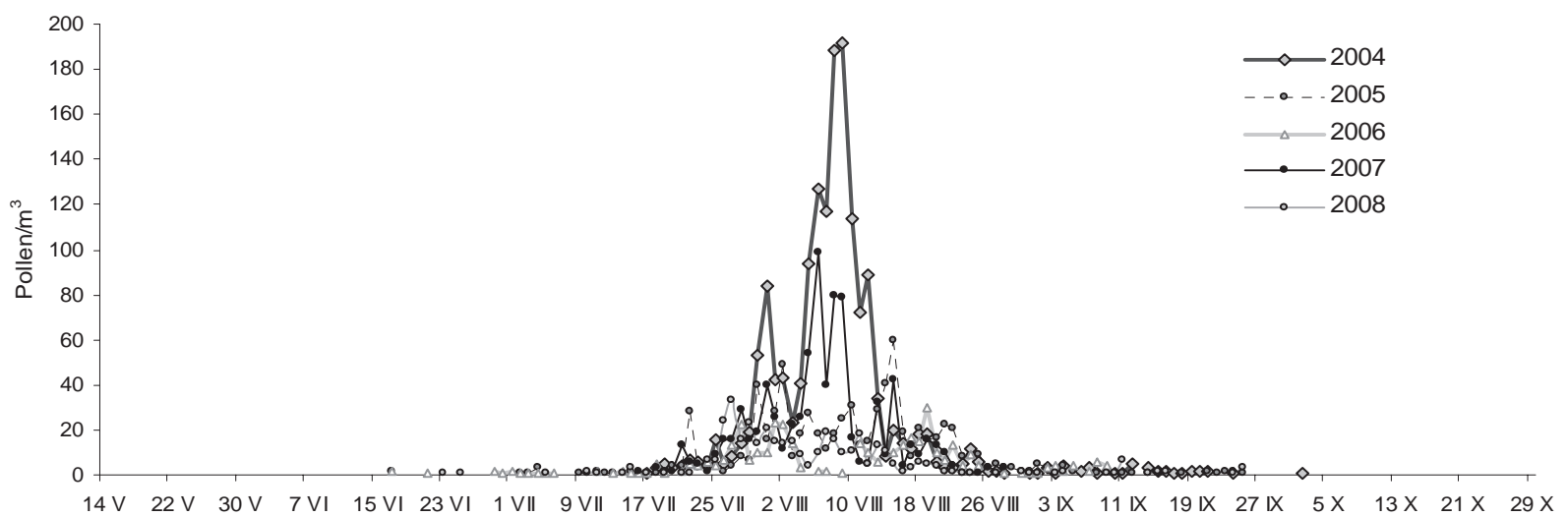

Fig. 4B. Seasonal values of Artemisia pollen count in Szczecin, 2004-2008. 

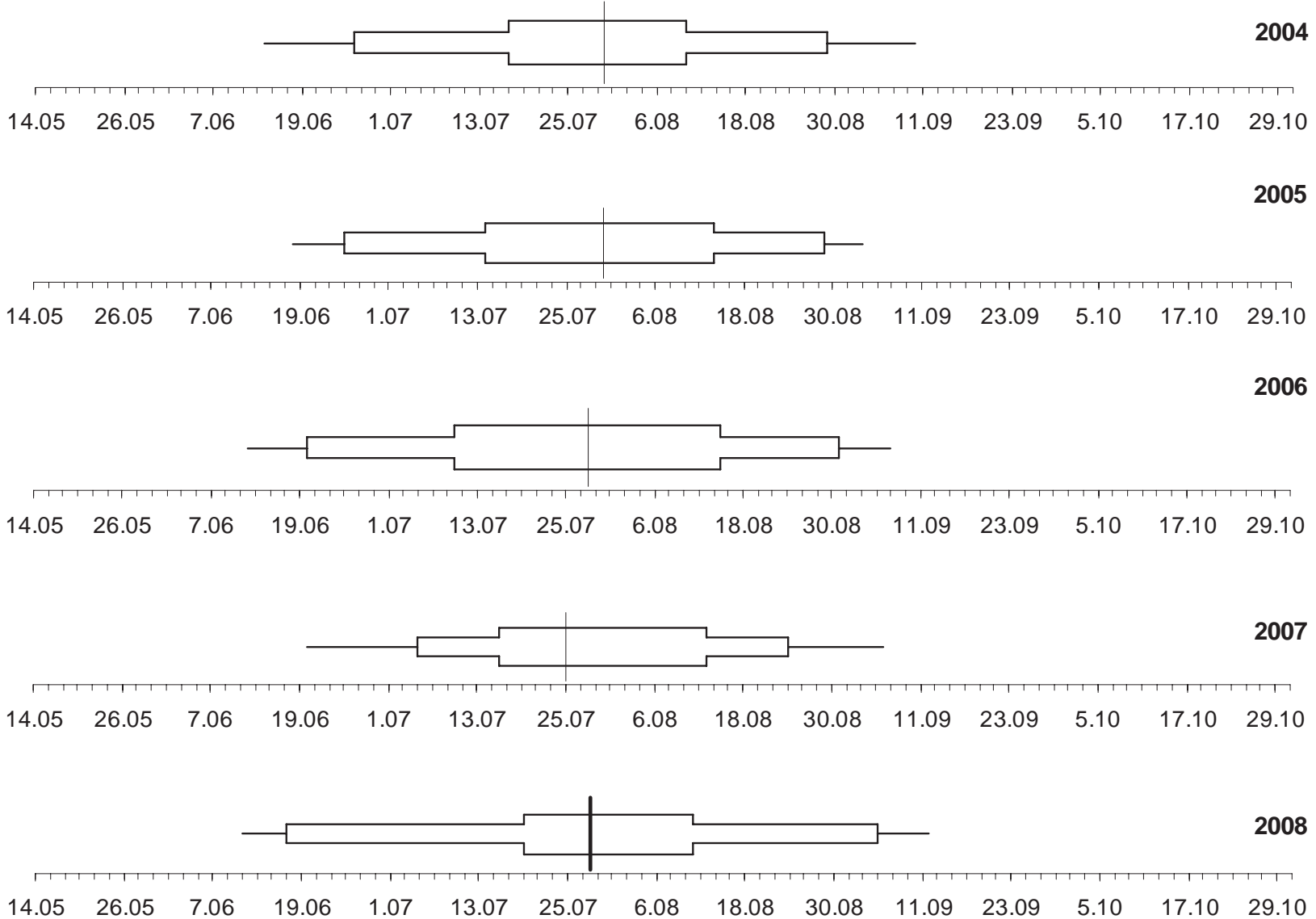

Fig. 5A. Dynamics of Urtica pollen seasons in Szczecin, 2004-2008. Diagrams of consecutive stages of the pollen season: 1\%, 5\%, $25 \%, 50 \%$ (vertical line), $75 \%, 95 \%, 99 \%$.

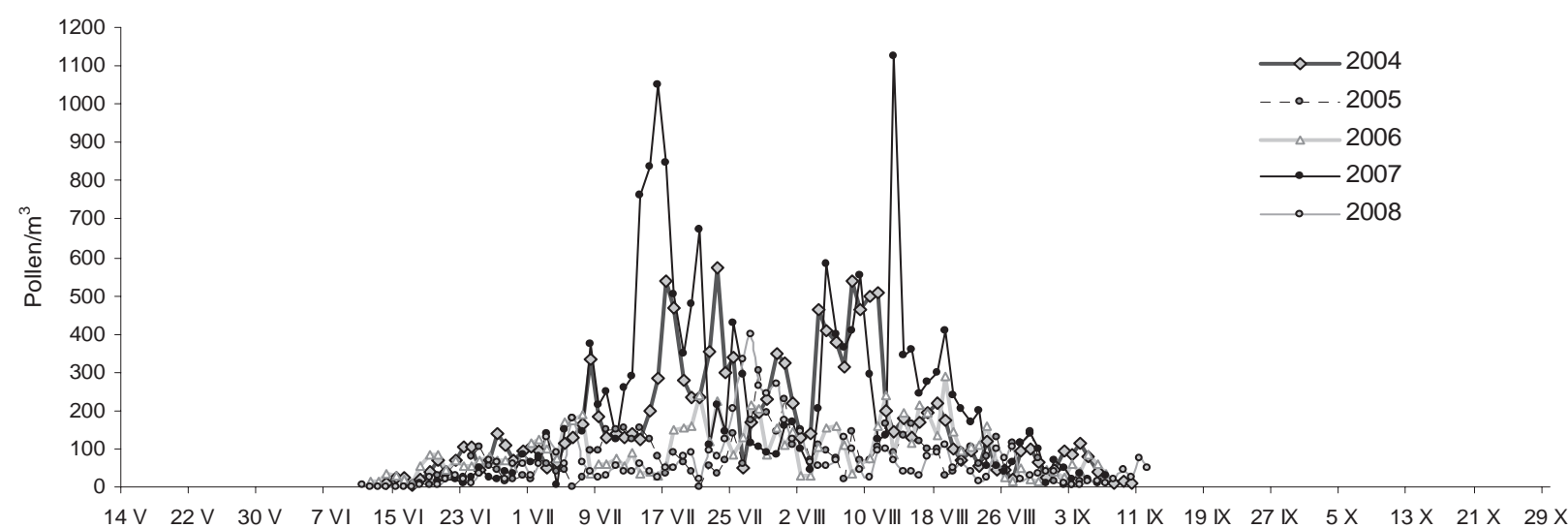

Fig. 5B. Seasonal values of Urtica pollen count in Szczecin, 2004-2008. 


\section{DISCUSSION}

The pollen presence in the air has been known to trigger allergic respiratory diseases. In summer, pollinosis is most often evoked by herbaceous taxa allergens. The Rumex, Plantago, Chenopodiaceae, Artemisia and Urtica sporomorphs occur abundantly in the air over the majority of the area of Poland from the end of May till the second decade of August ( $\mathrm{S} \mathrm{z} \mathrm{c} \mathrm{z} \mathrm{e} \mathrm{-}$ panek, 1994; Pi otrowska and WeryszkoChmielewska, 2003), which also holds true for Szczecin. Aerobiological literature provides different methods for determination of pollen seasons. The criteria for determination of the shortest and longest pollen seasons as well as the start and end dates, differ in various cities and for individual taxa under study. Often, the results for a given taxon also depended on the year of study. J a t o et al. (2006) analysed five pollen types (Betula, Poaceae, Olea, Platanus and Quercus) from three different areas (Ourence and Cordoba in Spain and Bologna in Italy) and observed the smallest differences in duration, start-dates and end-dates for Platanus and the greatest ones for Poaceae. In Szczecin, in the period of the 5 years of study the smallest differences in the dynamics of the pollen seasons were obtained for Urtica and Plantago, while significant differences were noted for Artemisia.

In Switzerland, the herbaceous species Plantago, Rumex and Urtica flower from the end of April to September, Chenopodiaceae from June to September and Artemisia from July to September. The seasonal pollen index (SPI) for these taxa is rather low and they play a minor role as allergy producers in Switzerland. In the years with hot, dry summers, a higher value than normal for the SPI is observed in the case of some of this species (G e h r i g , 2006), especially for Plantago and Chenopodiaceae. According to their ecological habitats, Plantago and Chenopodiaceae are more tolerant to drought than other species. Similar properties of the pollen season of these taxa were noted in Szczecin.

On the basis of analysis of the seasonal dynamics in the pollen calendars, S z c ze panek (1994), Kasprzyk (1995), Rodriguez-Rajo et al. (2003, 2004), J a to et al. (2006) and D o c a m p o et al. (2007) distinguished two groups of taxa with different courses of pollen seasons. The first group includes the taxa whose pollen season is characterised by compact single-peak seasons. The second group, comprising mainly herbaceous plants, includes the taxa in the rank of genus or family with many species (Rumex, Poaceae, Chenopodiaceae). These pollen seasons are long and show many peaks in successive years. Therefore, it can be concluded that the number of species included in the same pollen type significantly influ- ences the duration of the pollen season. The presence of many peaks on the diagrams illustrating the pollen seasons for this group of plants can be also related to variable weather conditions over a given area. An analogous character of the pollen seasons of the taxa studied was observed in Szczecin.

The dates of the initial phase and of the other phases of the pollen seasons of the taxa studied, noted in successive years, were different. The differences were accounted for by weather conditions, especially air temperature, relative humidity, rainfall and wind direction (M e s a et al. 2003, Silva Palacios et al. 2007). In Szczecin, the multiple regression analysis revealed strong correlations with relative humidity, maximum wind speed, maximum air temperature, dew point temperature for the Artemisia, Urtica and Plantago pollen seasons. Statistically significant Pearson's correlations were found mainly with relative humidity, air temperature and dew point. D o c a m o et al. (2007) also observed significant Spearman's correlations for Urticaceae pollen at minimum and mean temperature during the summer in Nerja, Spain. Similarly as in Szczecin, a positive, significant correlation between the Plantaginaceae pollen count and air temperature, dew point temperature and wind speed was noted in Sydney, Australia, by S t e n n e t and B e g g s (2004).

\section{CONCLUSIONS}

Analysis of the pollen seasons of selected herbaceous plants has shown that temporary differences in the dynamics of pollen seasons are most evident for Artemisia.

The taxa of long pollen seasons are usually characterised by low pollen annual totals. For the taxa showing short pollen seasons, particular phases are compact and the annual totals high.

The number of days with the threshold and high pollen count, important for pollinosis sufferers, was significant for Artemisia and Urtica.

The multiple regressions revealed strong correlations of the pollen count with relative humidity, maximum wind speed, maximum air temperature, dew point temperature for the Artemisia, Urtica and Plantago pollen seasons. Statistically significant Pearson's correlations were found mainly with relative humidity, air temperature and dew point.

\section{REFERENCES}

Docampo S., Recio M., Trigo M. M., Melgar M., Cabesudo B., 2007. Risk of pollen allergy in Nerja (southern Spain): a pollen calendar. Aerobiologia, 23: 189-199. 
D'A m at o G., S piek s m a F. T. M., 2004. Allergenic pollen in Europe. Grana, 30: 60-70.

Garcia-Mozo H., Dominguez-Vilches E., Galan C., 2007. Airborne allergenic pollen in natural areas: Hornachuelos Natural Park, Cordoba, Southern Spain. Ann. Agric. Environ Med., 14: 63-69. Aerobiologia, 22: 27-34.

Gehrig R., 2006. The influence of the hot and dry summer 2003 on the pollen seasons in Switzerland. Aerobiologia, 22: 27-34.

Jato V., Rodriquez-Rajo F. J., Alcazar P., De Nun$\mathrm{t} \mathrm{i}$ i s P., G al an C., 2006. May the definition of pollen season influence aerobiological results? Aerobiologia, 22: $13-25$.

Kadocsa E., Juhasz M., 2002. Study of airborne pollen composition and allergen spectrum of hay fever patients in South Hungary (1990-1999). Aerobiologia, 18: 203-209.

K a s pr zy k I., 1995. Palynological analyses of airborne pollen fall in Ostrowiec Świętokrzyski in 1995. Ann. Agric. Environ. Med., 3: 83-86.

Kasprzyk I., Uruska A., Szczepanek K., Latałowa M., Gaweł J., Harmata K., Myszkowska D., Stach., Stępalska D., 2004. Regional differentiation in the Dynamics of the pollen seasons of Alnus, Corylus and Fraxinus in Poland (Preliminary results). Aerobiologia, 20: 141-151.

Latałowa M., Miętus M., Urska A., 2002. Seasonal variations in the atmospheric Betula pollen count in Gdansk (Southern Baltic coast) in relation to meteorological parameters. Aerobiologia, 18: 33-43.

Mesa J. A. S., Smith M., Emberlin J., Allitt U., Caulton E., Gal an C., 2003. Characteristic of grass pollen seasons in area of southern Spain and the United Kingdom. Aerobiologia, 19: 243-250.

Nils son S., Pers on S., 1981. Tree pollen spectra in the Stockholm region (Sweden), 1973-1980. Grana, 20: 179-182.

Piotrowska K., Weryszko-Chmielewska E., 2003. Pollen count of selected taxa in the atmosphere of Lublin using two monitoring methods. Ann. Agric. Environ. Med., 10: 79-85.

Rapiejko P., Lipiec A., Wojdas A., Jurkiewicz D., 2004. Threshold pollen concentration necessary to evoke allergic symptoms. Int. Rev. of Allergology Clin. Immunol. 10/3: 91-94.

Rodriquez-Rajo F. J., Jato V., A ira J. M., 2003. Pollen content in the atmosphere of Lugo (NW Spain) with reference to meteorological factors (1999-2001). Aerobiologia, 19: 213-225.

Rodriquez-Rajo F. J., Iglesias I., Jato V., 2004. Allergenic airborne pollen monitoring of Vigo (NW Spain) in 1995-2001. Grana, 43: 164-173.

Silva Palacios I., Tormo Molina R., Munoz Rodriguez A. F., 2007. The importance of interactions between meteorological conditions when interpreting their effect on the dispersal for pollen from homogeneously distributed sources. Aerobiologia, 23: 17-26.
Spieksma F. T. M., Emberlin J., Hielmroos M., Jäger S., Leuschner L. M., 1995. Atmospheric birch (Betula) pollen in Europe: Trends and fluctuations in annual quantities and the staring dates of the season. Grana, 43: 51-57.

StatSoft, Inc. (2008). STATISTICA (data analysis software system), version 9.0. www.statsoft.com.

Stennet P. J., B egg s J., 2004. Pollen in the atmosphere of Sydney, Australia, and relationships with meteorological parameters. Grana, 42: 209-216.

Szczepanek K., 1994.. Pollen calendar for Cracow (South Poland) 1982-1991. Aerobiologia, 10: 65-75.

\section{Czynniki pogodowe \\ a dynamika sezonów pyłkowych wybranych taksonów roślin zielnych w Szczecinie, 2004-2008}

\section{Streszczenie}

Dynamika sezonów pyłkowych i czas trwania ich poszczególnych faz zależy od właściwości geobotanicznych regionu, rangi systematycznej badanego taksonu, klimatu, lokalizacji i obfitości źródeł pyłku. Celem pracy było porównanie dynamiki sezonów pyłkowych wybranych taksonów roślin zielnych w Szczecinie na tle warunków pogodowych. Badania prowadzono w latach 2004-2008. Pomiary stężenia pyłku wykonano metodą objętościową $\mathrm{z}$ zastosowaniem aparatu wolumetrycznego model Lanzoni VPPS 2000. Ziarna pyłku badanych roślin zielnych najobficiej występują w powietrzu latem, a termin początku sezonu pyłkowego i jego czas trwania wykazuje najsilniejszy związek z temperaturą i wilgotnością powietrza, słabszy z prędkością wiatru i temperaturą punktu rosy. Ocena tempa zmian stężenia pyłku w stosunku do całkowitej sumy rocznej wykazała największe zróżnicowanie dynamiki sezonu Artemisia. Sezony pyłkowe pozostałych analizowanych roślin zielnych wykazują podobieństwa odnośnie długości i dynamiki poszczególnych faz w latach. Taksony z wieloma gatunkami $\mathrm{w}$ randze rodzaju lub rodziny (Rumex, Plantago, Chenopodiaceae) nie mają wyraźnej fazy maksimum a sumy roczne pyłku są niskie. Dlatego też zagrożenie alergenami pyłku tych roślin było niewielkie i występowało jedynie w przypadku bylicy i pokrzywy. 
